\title{
DFT Studies of the Interactions of a Graphene Layer with Small Water Aggregates
}

\author{
R. R. Q. Freitas, ${ }^{+, \neq}$R. Rivelino, ${ }^{+, *}$ F. de Brito Mota, ${ }^{\dagger}$ and C. M. C. de Castilho ${ }^{+, \neq}$ \\ ${ }^{\dagger}$ Instituto de Física, Universidade Federal da Bahia, Campus Universitário da Federação, 40210-340 Salvador, Bahia, Brazil \\ ${ }^{\ddagger}$ Instituto Nacional de Energia e Ambiente-INCT-EA, Campus Universitário da Federação, Universidade Federal da Bahia, $40170-280$ \\ Salvador, Bahia, Brazil
}

ABSTRACT: We have investigated the structure, adsorption, electronic states, and charge transfer of small water aggregates on the surface of a graphene layer using density functional theory. Our calculations were focused on water adsorbates containing up to five water molecules interacting with one and both sides of a perfect freestanding sheet. Different orientations of the aggregates with respect to the graphene sites were considered. The results show that the adsorption energy of one water molecule is primarily determined by its orientation, although it is also strongly dependent on the implemented functional scheme. Despite its intrinsic difficulties with dispersion interactions, the Perdew and Wang's

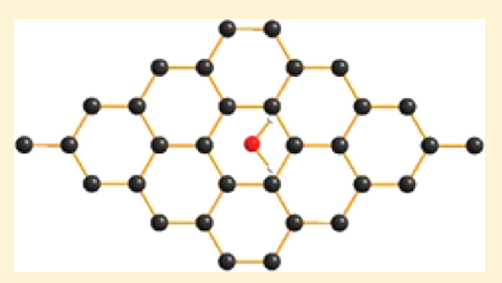
exchange-correlation functional may be a viable alternative to investigate the adsorption of large molecular aggregates on a graphene surface. Although water physisorption is expected to occur in the regime of droplets, we found no induced impurity states close to the Fermi level of graphene interacting with small water clusters. In order to investigate the donor/acceptor tendency of the water clusters on graphene, we have performed a Bader charge analysis. Considering the charge transfer mechanism, we have noticed that it should preferentially occur from water to graphene only when the oxygen atom is pointing toward the surface. Otherwise, and in the case of larger adsorbed clusters, charge transfers systematically occur from graphene to water.

\section{INTRODUCTION}

Studies of the effects of adsorbed molecules on the graphene surface, as well as their interactions through this monolayer of graphite, have open a new active research area aiming toward technological applications for a feasible two-dimensional membrane. $^{1-8}$ For example, an atomically thin coating of graphene has permitted to probe the microscopic structure of the first adlayer of water on mica under ambient conditions. ${ }^{1}$ Interestingly, tuning the electronic properties of pristine graphene by means of the adsorption of molecules on its surface is considered as one of the grand challenges for the development of graphene-based nanoelectronics. ${ }^{5-9}$ As is well-known, ${ }^{10}$ this one-atom-thick material exhibits a band structure with linear dispersion near the Fermi level, forming a zero-gap semiconductor. This of course limits the use of non doped freestanding graphene in electronic or photonic devices. However, the presence of adsorbate molecules could significantly change its conductivity, $^{11}$ even leading to an effective doping.

Among several possibilities ${ }^{12-15}$ of opening an energy gap in the band structure of graphene, water adsorbates have been proposed in the literature since its discovery. ${ }^{16-18}$ Recently, a tunable bandgap of up to $\sim 0.21 \mathrm{eV}$ has been obtained by Yavari et al., ${ }^{9}$ with a technique based on water adsorption to the graphene surface. This procedure has the advantage of being reversible and does not change chemically the graphene surface. However, these doping effects found experimentally are more complex mechanisms than single interactions of water molecules with graphene. Indeed, density-functional-theory (DFT) calculations have predicted that only water physisorption may induce impurity states close to the Fermi level. ${ }^{16}$ Hence, the presence of small aggregates adsorbed on graphene will not significantly change the electron mobility.

Although approaching a few molecules toward a graphene surface yields only a mild effect in its electron mobility, resistivity measurements in graphene have demonstrated a high sensitivity on detecting single molecules on its surface. ${ }^{7,11,14}$ Furthermore, preferential binding sites in graphene can favor the orientation and aggregation of molecules near the surface. ${ }^{19}$ Several theoretical works based on DFT calculations ${ }^{19-24}$ have aided to unveil the interaction energies of single molecules and small molecular aggregates with a graphene sheet. Most of these works have considered water clusters interacting with finite graphene flakes, ${ }^{20-24}$ for which the size effects on the calculated adsorption energy are rather important. ${ }^{23,24}$ Furthermore, in order to take into account the dispersion energy in these systems, approaches including an empirical van der Waals term are usually employed. Recently, correlated quantum-chemical treatments ${ }^{25}$ have been applied to investigate the adsorption of $\mathrm{H}_{2} \mathrm{O}$ on graphene.

In this work, we have performed first-principles DFT calculations to address the interactions of small water aggregates with a periodically extended graphene layer. Aiming to evaluate the performance of DFT in these calculations, two common functional schemes, which are not biased toward the description of intermolecular interactions, were employed. Also, the effects of

Received: June 2, 2011

Revised: September 26, 2011

Published: September 28, 2011 
using pseudopotentials on the structure and interaction of these adsorbates were carefully investigated. We have analyzed the adsorption energies of clusters containing up to five water molecules on a graphene surface. Also, we have focused on the interactions of water aggregates on both sides of this single membrane. Different orientations of the water clusters with respect to the graphene surface were considered. Also, charge transfers were calculated in order to investigate the donor/ acceptor character of these clusters on graphene, and our results were compared and discussed vis-à-vis with other theoretical results $^{19-24}$ and with experimental sets of water droplets on graphite, ${ }^{26}$ available in the literature.

\section{COMPUTATIONAL DETAILS}

The electronic and structural properties of our watergraphene and water-graphene-water systems were calculated by using the generalized gradient approximation (GGA) ${ }^{27-29}$ to the exchange correlation potential. The Kohn-Sham equations were solved using the Vienna $\mathrm{Ab}$ Initio Simulation Package $(\text { VASP })^{30-34}$ with the projector augmented wave (PAW) $)^{35,36}$ basis sets and $816 \mathrm{eV}$ as the plane wave cutoff. With this scheme, we have avoided the basis set superposition error (BSSE), commonly introduced by using localized basis sets in these types of systems. Moreover, ultrasoft pseudopotentials (USPP) $)^{30-37}$ were employed in order to evaluate the effects on the interaction energies of a single water molecule with a graphene sheet.

We have employed two standard schemes for the GGA that have been successfully used for the study of similar systems. $^{5,7,16,19}$ These are the Perdew-Burke-Ernzerhof $(\mathrm{PBE})^{28}$ and Perdew-Wang (PW91 ${ }^{29}$ approaches that are not biased toward the description of dispersion interactions. As it is known, GGA underestimates the binding energies as compared to the local density approximation (LDA), although the distances between the molecules and the surface are a little overestimated. ${ }^{7}$ However, if the system is found to be a bound complex with GGA it will be also bound within more sophisticated DFT approximations; e.g., including explicitly van der Waals interactions in the exchange-correlation potentials. ${ }^{38,39}$

For the adsorption of the water clusters (containing one up to five molecules), we considered a $4 \times 4$ graphene periodic supercell containing 32 atoms, with $16 \AA$ A between adjacent layers. Thus, we have avoided both edge and size effects. Besides that, this size for the supercell results as being a compromise between computational practical limits and the possibility of adsorption of small water aggregates. The resultant systems are of the types (i) $\left(\mathrm{H}_{2} \mathrm{O}\right)_{n} \cdots \mid$ and (ii) $\left(\mathrm{H}_{2} \mathrm{O}\right)_{n} \cdots \mid \cdot \cdot\left(\mathrm{H}_{2} \mathrm{O}\right)_{n}$, where the symbol "|" represents a freestanding graphene sheet and $n=1-5$. The total energy calculation and the structural relaxation were performed by sampling the Brillouin zone (BZ) with a $5 \times 5 \times 1$ Monkhorst-Pack $(\mathrm{MP})^{40}$ grid. Furthermore, the density of states (DOS) were calculated sampling the BZ with a $15 \times 15 \times 1 \mathrm{MP}$ grid and a Gaussian smearing of $0.14 \mathrm{eV}$. These values have been also chosen in ref 19. Additionally, charge transfers are calculated between the water aggregates and graphene by means of the Bader analysis ${ }^{41}$ on the charge density grid.

\section{WATER CLUSTERS ON GRAPHENE}

3.1. Benchmark Calculations for an Adsorbed Water Molecule. The calculated structure and dipole moment of the
Table 1. Structural Properties of the Isolated Water Molecule and Graphene Surface Calculated at Different Levels Of DFT ${ }^{a}$

\begin{tabular}{lcccc}
\multicolumn{1}{c}{ parameter } & PBE-PAW & PW91-PAW & PW91-USPP & expt./theory \\
$d_{\mathrm{OH}}(\AA), 42,43$ \\
$\theta_{\mathrm{HOH}}($ degree $)$ & 104.4 & 104.6 & 104.8 & 104.5 \\
$\mu(\mathrm{D})$ & 1.903 & 1.908 & 1.922 & 1.855 \\
$a(\AA)$ & 2.468 & 2.466 & 2.462 & 2.460 \\
$d_{\mathrm{CC}}(\AA)$ & 1.425 & 1.424 & 1.421 & 1.420
\end{tabular}

${ }^{a}$ Water: bond distance $\left(d_{\mathrm{OH}}\right)$, bond angle $\left(\theta_{\mathrm{HOH}}\right)$, and dipole moment $(\mu)$; graphene: bond distance $\left(d_{\mathrm{CC}}\right)$ and lattice constant $(a)$.

Table 2. Average Distances $(d)$ between the Water Molecule and the Graphene Surface, ${ }^{44}$ for Different Sites and Molecular Orientation, Calculated at Distinct Levels of DFT

\begin{tabular}{|c|c|c|c|c|c|}
\hline \multirow[b]{2}{*}{ position } & \multirow[b]{2}{*}{ orientation } & \multicolumn{4}{|c|}{$d(\AA)$} \\
\hline & & PBE-PAW & PW91-PAW & PW91-USPP & ${\mathrm{PBE}-\mathrm{TM}^{7}}^{7}$ \\
\hline B & $\mathrm{u}$ & 3.71 & 3.69 & 3.71 & 3.70 \\
\hline $\mathrm{T}$ & $\mathrm{u}$ & 3.71 & 3.69 & 3.71 & 3.70 \\
\hline $\mathrm{C}$ & $\mathrm{u}$ & 3.71 & 3.69 & 3.71 & 3.69 \\
\hline B & $\mathrm{n}$ & 3.55 & 3.58 & 3.54 & 3.55 \\
\hline $\mathrm{T}$ & $\mathrm{n}$ & 3.56 & 3.57 & 3.54 & 3.56 \\
\hline $\mathrm{C}$ & $\mathrm{n}$ & 3.55 & 3.55 & 3.54 & 3.55 \\
\hline B & $\mathrm{d}$ & 4.02 & 4.05 & 4.05 & 4.05 \\
\hline $\mathrm{T}$ & $\mathrm{d}$ & 4.02 & 4.05 & 4.07 & 4.05 \\
\hline $\mathrm{C}$ & $\mathrm{d}$ & 4.02 & 4.05 & 4.08 & 4.02 \\
\hline
\end{tabular}

water molecule and the lattice constant of the graphene cell are reported in Table 1. The results obtained with the different DFT approaches used here are compared to reliable reference values for these systems. ${ }^{10,42,43}$ As can be seen in Table 1, the use of USPP with PW91 leads to values slightly larger than those calculated with PAW for the water properties. However, the calculated values of the lattice constant and carbon-carbon bonds in graphene are slightly smaller using USPP. A similar pattern is noticed by comparing PBE to PW91 using PAW. We have examined diverse orientations of $\mathrm{H}_{2} \mathrm{O}$ relative to the graphene layer, in accordance with ref 7 , within the present levels of calculations. Thus, we have considered the adsorption sites on top of a carbon atom (T), on the hexagon center (C), and in the center of a carbon-carbon bond (B). From these positions, the distances of a water molecule to the layer were also calculated. ${ }^{44}$

The orientation of the water molecule with respect to the surface is chosen as follows: $\mathrm{O}-\mathrm{H}$ bonds up (u), down (d), or parallel $(\mathrm{n})$ to the graphene surface. ${ }^{7}$ Our calculated distances, in comparison to recent values, are given in Table 2. The present results indicate that this property presents a very small sensitivity to the functional method with PAW, varying in a $0.01-0.03 \AA$ range. The effect of USPP in PW91 is also mild on the calculated distances. Moreover, for each site on the graphene sheet and orientation of $\mathrm{H}_{2} \mathrm{O}$, we have calculated the adsorption energy, defined as $E_{a d s}=E_{G}+E_{\mathrm{H} 2 \mathrm{O}}-E_{t o t}$, where $E_{G}$ is the energy of a pristine graphene layer, $E_{\mathrm{H} 2 \mathrm{O}}$ is the energy of a relaxed water molecule, and $E_{t o t}$ is the total energy of the relaxed $\mathrm{H}_{2} \mathrm{O} \cdots$ | system. Thus, the energy interaction of the molecular adsorbate was calculated with the different level of approximations considered here. 
Table 3. Adsorption Energies $\left(E_{\text {ads }}\right)$ of One Water Molecule on Graphene, For Different Sites and Molecular Orientations, Calculated at Distinct Levels of DFT

\begin{tabular}{|c|c|c|c|c|c|}
\hline \multirow[b]{2}{*}{ position } & \multirow[b]{2}{*}{ orientation } & \multicolumn{4}{|c|}{$E_{\mathrm{ads}}(\mathrm{meV})$} \\
\hline & & PBE-PAW & PW91-PAW & PW91-USPP & PBE-TM $^{7}$ \\
\hline B & $\mathrm{u}$ & 21 & 33 & 74 & 18 \\
\hline $\mathrm{T}$ & $\mathrm{u}$ & 21 & 34 & 74 & 19 \\
\hline $\mathrm{C}$ & $\mathrm{u}$ & 22 & 35 & 74 & 20 \\
\hline B & $\mathrm{n}$ & 28 & 38 & 80 & 24 \\
\hline $\mathrm{T}$ & $\mathrm{n}$ & 25 & 37 & 82 & 24 \\
\hline $\mathrm{C}$ & $\mathrm{n}$ & 26 & 40 & 83 & 27 \\
\hline B & $\mathrm{d}$ & 21 & 32 & 73 & 18 \\
\hline $\mathrm{T}$ & $\mathrm{d}$ & 21 & 32 & 73 & 19 \\
\hline $\mathrm{C}$ & $\mathrm{d}$ & 22 & 33 & 72 & 19 \\
\hline
\end{tabular}
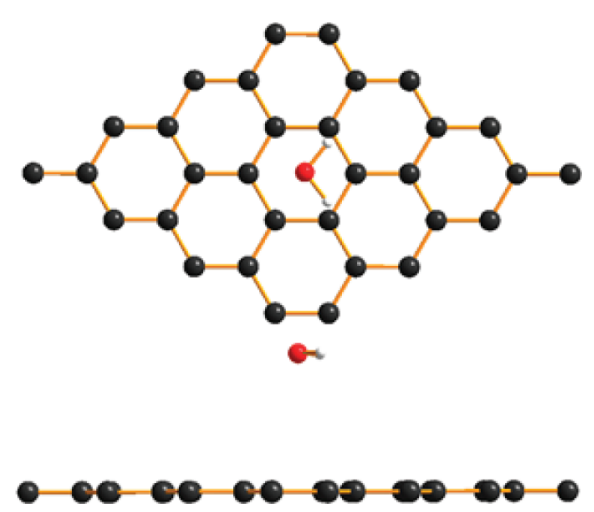

Figure 1. Structure of a water molecule adsorbed on a $4 \times 4$ supercell of graphene. View from the top and side of the parallel (n) orientation on a C site.

In Table 3, we report the calculated adsorption energies compared to other DFT calculations. As expected for these methods, the adsorption energies are small though sufficient to determine the most favorable position and orientation of $\mathrm{H}_{2} \mathrm{O}$ relatively to the graphene layer (Figure 1). Furthermore, we notice that the calculated values clearly depend on the GGA scheme as well as on the pseudopotentials utilized. For example, using PW91-USPP we obtain adsorption energies more than twice as compared to PW91-PAW. Additionally, most of the values obtained within PW91-PAW are larger than 50\% as compared to the PBE-PAW values, except for $\mathrm{H}_{2} \mathrm{O}$ in $\mathrm{n}$ orientations on $\mathrm{B}$ or $\mathrm{T}$ site of graphene. However, our $\mathrm{PBE}$ adsorption energies are more similar to those values obtained using pseudopotentials of the Troullier-Martins type. ${ }^{7}$ In general, all of these calculations show that the adsorption energy is much more determined by the water orientation than by the site position on graphene.

The calculated dissociation curves of the $\mathrm{H}_{2} \mathrm{O} \cdots \mid$ system in the $\mathrm{u}$-C geometry are displayed in Figure 2 for the different DFT methods. Our PBE-PAW curve gives a shallow dip to a minimum with adsorption energy of $22 \mathrm{meV}$ and goes to zero for distances larger than $5 \AA$. This minimum is dipper $(35 \mathrm{meV})$ when we have employed PW91-PAW, but the curve goes to zero for distances larger than $5.5 \AA$. Interestingly, a minimum value of $74 \mathrm{meV}$ is obtained by using the PW91 functional combined with USPP. We note that the PW91-USPP adsorption energy is quite similar

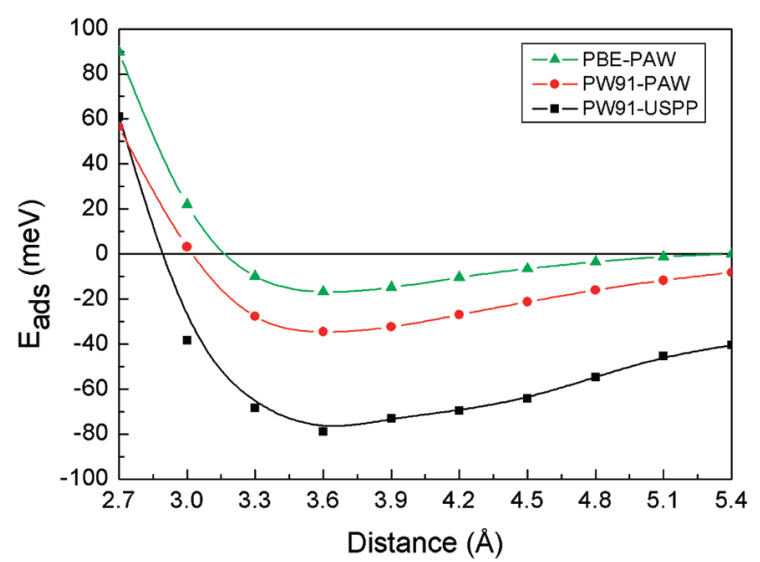

Figure 2. Calculated dissociation curves with different DFT schemes for the $\mathrm{H}_{2} \mathrm{O}$-graphene system (from the $\mathrm{u}$ - $\mathrm{C}$ geometry).

Table 4. Charge Transfers $(\Delta Q)$ from Graphene to the Water Molecule, For Different Sites and Molecular Orientation, Within the Bader Analysis

\begin{tabular}{|c|c|c|c|c|c|}
\hline \multirow[b]{2}{*}{ position } & \multirow[b]{2}{*}{ orientation } & \multicolumn{4}{|c|}{$\Delta Q(\mathrm{e})$} \\
\hline & & PBE-PAW & PW91-PAW & PBE-TM $^{7}$ & PBE-TM ${ }^{19}$ \\
\hline B & $\mathrm{u}$ & -0.0015 & -0.0010 & -0.021 & \\
\hline $\mathrm{T}$ & $\mathrm{u}$ & -0.0012 & -0.0014 & -0.021 & \\
\hline $\mathrm{C}$ & $\mathrm{u}$ & -0.0009 & -0.0010 & -0.021 & -0.0028 \\
\hline B & $\mathrm{n}$ & -0.0020 & -0.0016 & -0.013 & \\
\hline $\mathrm{T}$ & $\mathrm{n}$ & -0.0016 & -0.0017 & -0.015 & \\
\hline $\mathrm{C}$ & $\mathrm{n}$ & -0.0026 & -0.0025 & -0.014 & \\
\hline B & $\mathrm{d}$ & 0.0007 & 0.0000 & 0.009 & \\
\hline $\mathrm{T}$ & $\mathrm{d}$ & 0.0011 & -0.0002 & 0.009 & \\
\hline $\mathrm{C}$ & $\mathrm{d}$ & 0.0006 & 0.0006 & 0.010 & \\
\hline
\end{tabular}

to the value of $83 \mathrm{meV}$ calculated in ref 25 , by using the PBE scheme including a semiempirical dispersion correction term. However, the limit of the dissociation curve obtained with PW91-USPP is very far from 5.5 ̊. Although we have calculated adsorption energy values of the order of $83 \mathrm{meV}$ (for the $\mathrm{n}-\mathrm{C}$ geometry), the PW91-USPP values appear to underestimate accurate quantum-chemistry calculations. ${ }^{22,25}$

Indeed, most of the more accurate works calculating the adsorption energy of water on graphene have used fused-benzene ring models to address the problem..$^{20-24}$ Considering large graphene flakes interacting with a water molecule, the binding energy was estimated to be in a range of 126 to $154 \mathrm{meV}$, with DFT calculations including dispersion interaction terms. Also, these values were obtained for a d-C type geometry (see the present nomenclature in Table 3). In our case, the more energetically favorable geometry configuration for the $\mathrm{H}_{2} \mathrm{O} \cdots$ | system was the $\mathrm{n}-\mathrm{C}$ type. Curiously, values obtained in ref 22 for $\mathrm{n}-\mathrm{C}$ and $\mathrm{u}-\mathrm{C}$ geometries were, respectively, 90 and $50 \mathrm{meV}$, which seem to be too small if compared to the adsorption energy (154 meV) of the d-C geometry. For instance, according to recent $\operatorname{CCSD}(\mathrm{T})$ calculations, ${ }^{25}$ on going from the $\mathrm{u}-\mathrm{C}$ structure $(108 \mathrm{meV})$ to the d-C structure $(123 \mathrm{meV})$, one obtains an increment of $\sim 14 \%$ in the adsorption energy. Similarly, we have obtained an increment of $\sim 12 \%$ (PW91-USPP) considering these structures, although our absolute values lack long-range 
electron correlation. In fact, our calculated dependence on the water orientation, regarding distinct adsorption sites, gives small variations in the adsorption energy (Table 3 ).

Despite the difficulties in describing the dispersion interaction with standard DFT, the PW91 functional may be a viable alternative to investigate the adsorption of very large clusters on a graphene surface. For instance, recent measurements ${ }^{26}$ of the water-graphite surface interaction have demonstrated that the adsorption energy is of 79.1 and $74.4 \mathrm{meV}$, obtained for contact angles of $42^{\circ}$ and $86^{\circ}$, respectively. Thus, considering $\sim 50 \%$ loss in the interaction energy, the combination of PW91 with the PAW basis sets offers an interesting method to perform first-principles calculations for water-graphene systems. As is known, PW91 is one of the best compromises among the standard GGA functionals for calculations of weakly bound

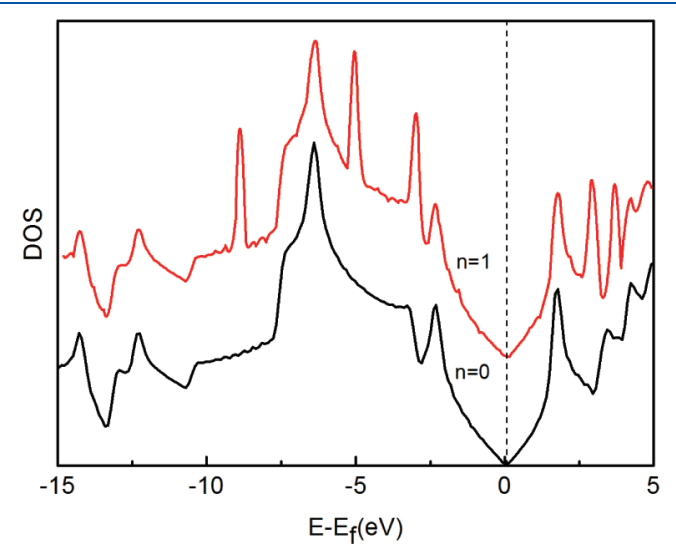

Figure 3. Calculated density of states (DOS) of a graphene sheet: isolated and containing one $\mathrm{H}_{2} \mathrm{O}$ adsorbed (n orientation on a $\mathrm{B}$ site). complexes. ${ }^{45}$ It is also important to notice that the appropriate description of the surface (by considering periodic supercells instead of a graphene flake) appears to significantly influence the description of the systems.

Using the Bader charge analysis method combined with the PAW scheme, we have evaluated the possible charge transfer (CT) between graphene and the water molecule. The results are presented in Table 4. We noticed that a small CT from the water molecule to graphene occurs only for the molecule in $\mathrm{u}$ or $\mathrm{n}$ orientation. On the other hand, when the water molecule is in $\mathrm{d}$ orientation, the tendency of CT is from graphene. As can be seen, the CT gives only a mild value that is partially due to the calculation method. The largest CT from $\mathrm{H}_{2} \mathrm{O}$ to graphene is approximately $0.003 e$ (see Table 4) with either PW91 or PBE, for the water molecule in $\mathrm{n}$ orientation on the $\mathrm{C}$ site. Although the CT values are practically independent of the adsorption site, we notice here a CT tendency from graphene to $\mathrm{H}_{2} \mathrm{O}$ with $\mathrm{PBE}$ for the water molecule adsorbed in $\mathrm{d}$ orientation. A similar inversion was also observed in ref 7 based on the Hirshfeld charge analysis. However, using the PW91 charge density, no CT is expected on B or $\mathrm{T}$ sites. Indeed, in the regime of a few water molecules on graphene, CT should be a very small quantity, since a single water molecule cannot induce impurity states close to the Fermi level (see the calculated DOS in Figure 3). We will discuss further these effects for larger water adsorbates.

3.2. Structure, Energetics and Charge Transfer of Larger Water Clusters. Now we examine the properties of small water aggregates containing up to five molecules adsorbed on the graphene surface, i.e., the $\left(\mathrm{H}_{2} \mathrm{O}\right)_{n} \cdots \mid$ systems, with $n=2-5$ (see Figure 4$)$. We notice that among our selected clusters the dimer presents the largest dipole moment (about 2.73 D with $\mathrm{PBE}$ ), while the other clusters present a dipole moment smaller than the isolated water molecule (see the calculated values in

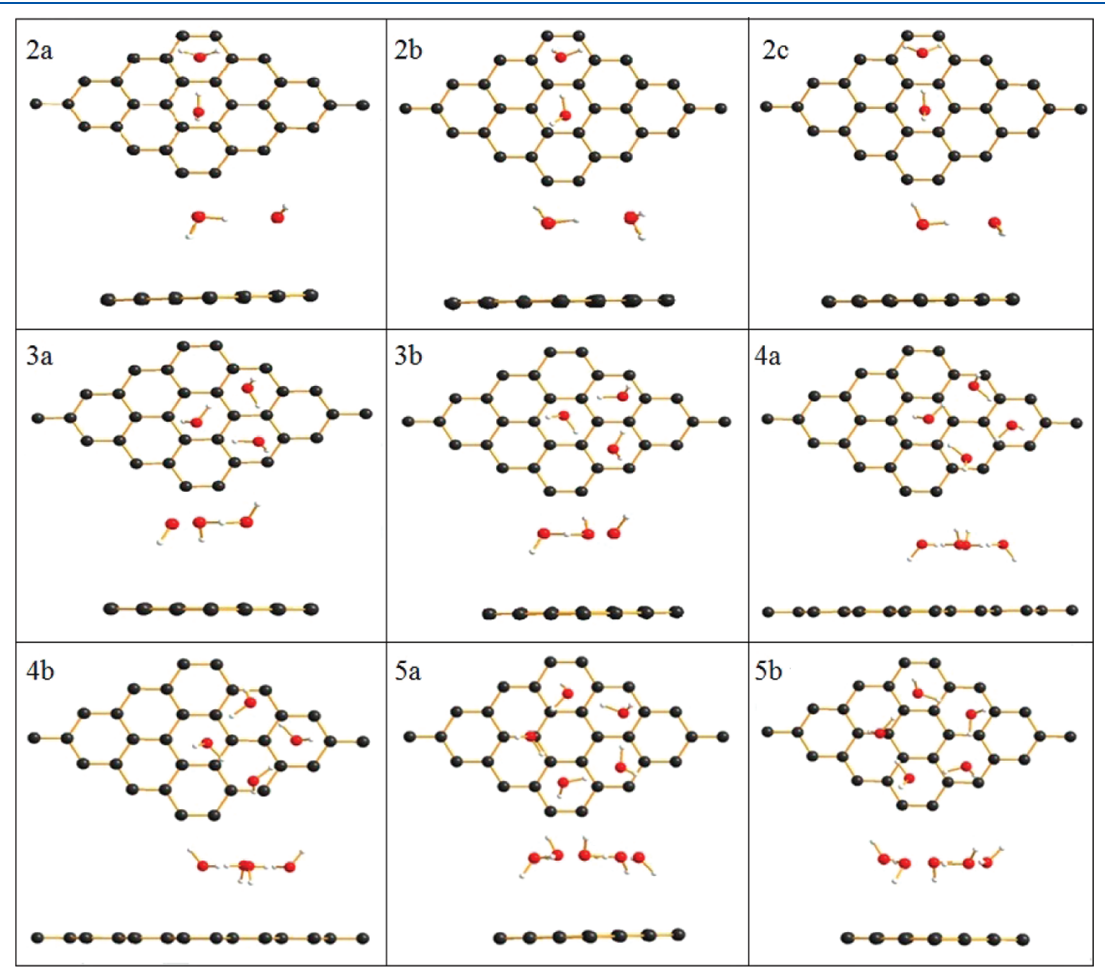

Figure 4. Structures of different water clusters adsorbed on graphene. View from the top and side of all of the different adsorbates on the graphene surface. 
Table 5). These small values of dipole moments are important to avoid artificial dipole-dipole interactions due to the size of the supercell. We have considered the most stable water clusters, ${ }^{19,43}$ which are fully relaxed as (i) isolated and (ii) on the graphene sheet. After full geometry optimization procedures, we have calculated the adsorption energy, i.e., $E_{\mathrm{ads}}=E_{\mathrm{G}}+E_{\text {cluster }}-E_{\text {tot }}$, where $E_{\mathrm{G}}$ is the energy of a pristine graphene layer, $E_{\text {cluster }}$ is the energy of the relaxed aggregate in vacuum, and $E_{\text {tot }}$ is the total energy of the relaxed $\left(\mathrm{H}_{2} \mathrm{O}\right)_{n} \cdots \mid$ systems.

The calculated adsorption energies are presented in Table 6. In agreement with ref 19 , we find three distinct orientations for the water dimer with respect to the graphene surface (structures $2 a-c$ in Figure 4). Additionally, we have considered two possible orientations for the trimer, tetramer, and pentamer (structures 3a, 3b, 4a, 4b, 5a, and 5b in Figure 4). All of these structures are weakly bound to the graphene surface with adsorption energy per molecule in the 14-29 meV range with PBE-PAW and 19$40 \mathrm{meV}$ range with PW91-PAW. These values do not depend

Table 5. Dipole Moments $(\boldsymbol{\mu})$ of the Isolated Water Clusters Calculated at Different Levels of DFT

\begin{tabular}{lccc} 
& \multicolumn{3}{c}{$\mu(\mathrm{D})$} \\
\cline { 2 - 4 } cluster & PBE-PAW & PW91-PAW & ref 43 \\
\hline dimer & 2.729 & 2.752 & 2.683 \\
trimer & 1.194 & 1.195 & 1.071 \\
tetramer & 0.000 & 0.000 & 0.000 \\
pentamer & 1.027 & 1.031 & 0.927
\end{tabular}

significantly on the orientation and size of the cluster with respect to the graphene sheet, except for $\left(\mathrm{H}_{2} \mathrm{O}\right)_{2} \cdots \mid$ (structure $2 \mathrm{a}$ in Figure 4 ) that presents adsorption energy $\sim 50 \%$ smaller than the other orientations of the dimer ( $2 b$ and $2 c)$. On the other hand, it is also known that the binding energy of water aggregates with a graphene surface depend on the number of water molecules which form hydrogen bonds. ${ }^{20}$

The highest total adsorption energy calculated in the present work was for pentamer $5 \mathrm{~b}$ (99 meV with PW91). However, as a result of the number of hydrogen bonds formed in the cluster structure, the corresponding energy per molecule was of about $20 \mathrm{meV}$. Indeed, in order to have a better description of the water-graphene surface interactions, it would be necessary to consider a large coverage of water adsorbates. ${ }^{26}$ As it is expected, the adsorption energy tends to higher values by increasing the number of water molecules in the aggregate, being also dependent on the structure of each aggregate. For these adsorbates, the best agreement of our PBE results with those from ref 19 is for dimer $2 \mathrm{c}$. Also, the values obtained for the two trimer ( $3 \mathrm{a}$ and $3 \mathrm{~b}$ ) and for the two pentamer ( $5 \mathrm{a}$ and $5 \mathrm{~b}$ ) orientations are in line with the previous calculations. In the case of the orientation of the water dimer $2 b$, we have obtained the largest difference in the adsorption energy calculated at PBE. In fact, there are many local minima on the graphene surface and the exact orientation of the dimer is much more sensitive to the position of the adsorption sites than in the case of larger aggregates.

Analyzing the charge transfers between the water aggregates and graphene, we notice a significant increase of this quantity in the largest clusters. For example, in the case of the water dimer

Table 6. Total Adsorption Energies $\left(E_{\text {ads }}\right)$ and Their Averages Per Molecule for Clusters with Different Sizes and Orientations

\begin{tabular}{|c|c|c|c|c|c|c|c|}
\hline \multirow[b]{2}{*}{ no. of $\mathrm{H}_{2} \mathrm{O}$ mol. } & \multirow[b]{2}{*}{ orientation } & \multicolumn{2}{|c|}{ PBE-PAW } & \multicolumn{2}{|c|}{ PW91-PAW } & \multicolumn{2}{|c|}{ PBE-TM ${ }^{19}$} \\
\hline & & $E_{\text {ads }}(\mathrm{meV})$ & $E_{\mathrm{ads}} / \mathrm{mol} .(\mathrm{meV})$ & $E_{\text {ads }}(\mathrm{meV})$ & $E_{\text {ads }} / \mathrm{mol} .(\mathrm{meV})$ & $E_{\text {ads }}(\mathrm{meV})$ & $E_{\mathrm{ads}} / \mathrm{mol} .(\mathrm{meV})$ \\
\hline 2 & $a$ & 32 & 16 & 50 & 25 & 28 & 14 \\
\hline 2 & $\mathrm{~b}$ & 58 & 29 & 80 & 40 & 65 & 32 \\
\hline 2 & c & 58 & 29 & 76 & 38 & 56 & 28 \\
\hline 3 & a & 53 & 18 & 74 & 25 & 48 & 16 \\
\hline 3 & $\mathrm{~b}$ & 54 & 18 & 76 & 25 & 49 & 16 \\
\hline 4 & a & 57 & 14 & 81 & 20 & 52 & 13 \\
\hline 4 & $\mathrm{~b}$ & 63 & 16 & 88 & 22 & & \\
\hline 5 & a & 68 & 14 & 96 & 19 & 64 & 13 \\
\hline 5 & $\mathrm{~b}$ & 70 & 14 & 99 & 20 & 65 & 13 \\
\hline
\end{tabular}

Table 7. Charge Transfers $(\Delta Q)$ from Graphene to the Water Aggregates and Their Averages Per Molecule for Clusters with Different Sizes and Orientations

\begin{tabular}{|c|c|c|c|c|c|c|c|}
\hline \multirow[b]{2}{*}{ no. of $\mathrm{H}_{2} \mathrm{O}$ mol. } & \multirow[b]{2}{*}{ orientation } & \multicolumn{2}{|c|}{ PBE-PAW } & \multicolumn{2}{|c|}{ PW91-PAW } & \multicolumn{2}{|c|}{ PBE-TM ${ }^{19}$} \\
\hline & & $\Delta Q(\mathrm{e})$ & $\Delta Q /$ mol. (e) & $\Delta Q(\mathrm{e})$ & $\Delta Q / \mathrm{mol}$. (e) & $\Delta Q(\mathrm{e})$ & $\Delta Q / \mathrm{mol}$. (e) \\
\hline 2 & $\mathrm{a}$ & 0.0030 & 0.0015 & 0.0031 & 0.0015 & 0.0026 & 0.0013 \\
\hline 2 & $\mathrm{~b}$ & 0.0052 & 0.0026 & 0.0055 & 0.0026 & 0.0114 & 0.0057 \\
\hline 2 & $\mathrm{c}$ & 0.0043 & 0.0021 & 0.0042 & 0.0021 & 0.0073 & 0.0036 \\
\hline 3 & $\mathrm{a}$ & 0.0052 & 0.0017 & 0.0049 & 0.0016 & 0.0038 & 0.0013 \\
\hline 3 & $\mathrm{~b}$ & 0.0035 & 0.0012 & 0.0032 & 0.0010 & 0.0114 & 0.0038 \\
\hline 4 & $\mathrm{a}$ & 0.0068 & 0.0017 & 0.0068 & 0.0017 & 0.0058 & 0.0014 \\
\hline 4 & $\mathrm{~b}$ & 0.0081 & 0.0020 & 0.0083 & 0.0021 & - & - \\
\hline 5 & $\mathrm{a}$ & 0.0112 & 0.0022 & 0.0115 & 0.0023 & 0.0065 & 0.0013 \\
\hline 5 & $\mathrm{~b}$ & 0.0110 & 0.0022 & 0.0106 & 0.0021 & 0.0094 & 0.0019 \\
\hline
\end{tabular}




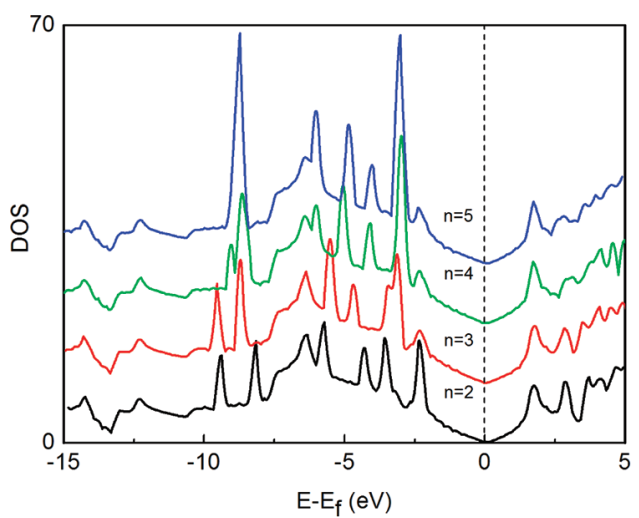

Figure 5. Calculated density of states (DOS) of different water clusters on graphene in the most stable orientations. The number of water molecules in the cluster is denoted by $n$.

and trimer we have calculated the CT values (from graphene to the water molecules) between 0.003 and $0.005 e$ with either PBE or PW91 (Table 7). However, in the case of the tetramer the CT increases to $0.007-0.008 e$, while in the case of the pentamer the CT increases to $0.01 \mathrm{e}$. This can indicate that the larger the adsorbed water aggregate, the larger the CT from the graphene surface. Thus, a large amount of water adsorbed to the graphene surface could affect the charge carrier concentration., ${ }^{9,16}$ Our calculated CT values are also in line with the Hirshfeld charge analysis method from ref 19. In the limit of small clusters, however, there is no significant change in the density of states close to the Fermi level, as displayed in Figure 5. Furthermore, as discussed previously for the adsorbed monomer, the Bader charge analysis associated to the GGA schemes leads to very small CT values.

\section{WATER CLUSTER INTERCALATED BY A GRAPHENE SHEET}

4.1. Adsorption Energy. By means of PW91 and PBE DFT methods, it is also useful to evaluate the adsorption energy of water aggregates adsorbed on both sides of a graphene sheet. Thus, we present results for systems of the type $\left(\mathrm{H}_{2} \mathrm{O}\right)_{n} \cdots$ | $\cdots\left(\mathrm{H}_{2} \mathrm{O}\right)_{n}$, with $n=1-5$. The starting points for the relaxation process consider, as average distance, between the two water aggregates an amount of $\sim 7 \AA$. The optimized structures are displayed in Figure 5. We have calculated the adsorption energies of these molecular adsorbates as defined in Sec. 3. Our results obtained with both functionals, using PAW, are presented in Table 8. Considering now the three orientations $(\mathrm{u}, \mathrm{d}, \mathrm{n})$ of a water molecule in $\mathrm{H}_{2} \mathrm{O} \cdots \mid \cdot \cdot \mathrm{H}_{2} \mathrm{O}$, we have noticed that the structure with $\mathrm{n}$ orientation (1a) presents an adsorption energy a little smaller than its corresponding structure interacting with only one side of graphene (see Figure 1). This finding can indicate that the interaction of water molecules intercalated by a graphene sheet also depends on the relative dipole orientation, since this structure represents the most repulsive dipole configuration. This effect is however lessened in the case of the other two configurations ( $1 \mathrm{~b}$ and $1 \mathrm{c}$ ), which give adsorption energies very similar to the case of one-side adsorbed water molecule.

We have also observed an important effect of the dipole component in the case of the $\left(\mathrm{H}_{2} \mathrm{O}\right)_{2} \cdots \mid \cdot \cdot\left(\mathrm{H}_{2} \mathrm{O}\right)_{2}$ systems. As given in Table 8 , structure 2a presents the smallest value for the adsorption energy ( $94 \mathrm{meV}$ with PW91) in comparison to
Table 8. Total Adsorption Energies $\left(E_{a d s}\right)$ and Their Averages Per Molecule for the Intercalated Clusters with Different Sizes and Orientations

\begin{tabular}{|c|c|c|c|c|c|}
\hline \multirow[b]{2}{*}{ no. of $\mathrm{H}_{2} \mathrm{O}$ mol. } & \multirow[b]{2}{*}{ orientation } & \multicolumn{2}{|c|}{ PBE-PAW } & \multicolumn{2}{|c|}{ PW91-PAW } \\
\hline & & $\begin{array}{c}E_{\text {ads }} \\
(\mathrm{meV})\end{array}$ & $\begin{array}{c}E_{\mathrm{ads}} / \mathrm{mol} . \\
(\mathrm{meV})\end{array}$ & $\begin{array}{c}E_{\text {ads }} \\
(\mathrm{meV})\end{array}$ & $\begin{array}{c}E_{\mathrm{ads}} / \mathrm{mol} . \\
(\mathrm{meV})\end{array}$ \\
\hline 1 & a & 47 & 23 & 70 & 35 \\
\hline 1 & c & 49 & 24 & 72 & 36 \\
\hline 1 & c & 48 & 24 & 70 & 35 \\
\hline 2 & $\mathrm{a}$ & 64 & 16 & 94 & 24 \\
\hline 2 & $\mathrm{~b}$ & 139 & 35 & 170 & 42 \\
\hline 2 & $\mathrm{c}$ & 130 & 32 & 168 & 42 \\
\hline 3 & $\mathrm{a}$ & 111 & 18 & 154 & 26 \\
\hline 3 & $b$ & 106 & 18 & 148 & 25 \\
\hline 4 & $\mathrm{a}$ & 116 & 14 & 168 & 21 \\
\hline 4 & $\mathrm{~b}$ & 113 & 14 & 168 & 21 \\
\hline 5 & a & 129 & 13 & 177 & 18 \\
\hline 5 & $\mathrm{~b}$ & 100 & 10 & 157 & 16 \\
\hline
\end{tabular}

Table 9. Charge Transfers $(\Delta Q)$ from Graphene to the Water Aggregates and Their Averages Per Molecule for the Intercalated Clusters with Different Sizes and Orientations

\begin{tabular}{|c|c|c|c|c|c|}
\hline \multirow{2}{*}{$\begin{array}{c}\text { no. of } \\
\mathrm{H}_{2} \mathrm{O} \text { mol. }\end{array}$} & \multirow[b]{2}{*}{ orientation } & \multicolumn{2}{|c|}{ PBE-PAW } & \multicolumn{2}{|c|}{ PW91-PAW } \\
\hline & & $\Delta Q(\mathrm{e})$ & $\Delta Q /$ mol. (e) & $\Delta Q(\mathrm{e})$ & $\Delta Q / \mathrm{mol}$. (e) \\
\hline 1 & a & -0.0052 & -0.0026 & -0.0052 & -0.0026 \\
\hline 1 & $\mathrm{~b}$ & -0.0008 & -0.0004 & -0.0010 & -0.0005 \\
\hline 1 & c & 0.0007 & 0.0003 & 0.0005 & 0.0002 \\
\hline 2 & $\mathrm{a}$ & 0.0048 & 0.0012 & 0.0046 & 0.0012 \\
\hline 2 & $\mathrm{~b}$ & 0.0096 & 0.0024 & 0.0096 & 0.0024 \\
\hline 2 & $\mathrm{c}$ & 0.0078 & 0.0020 & 0.0062 & 0.0016 \\
\hline 3 & $\mathrm{a}$ & 0.0086 & 0.0014 & 0.0086 & 0.0014 \\
\hline 3 & $\mathrm{~b}$ & 0.0095 & 0.0016 & 0.0047 & 0.0008 \\
\hline 4 & $\mathrm{a}$ & 0.0142 & 0.0018 & 0.0141 & 0.0017 \\
\hline 4 & $\mathrm{~b}$ & 0.0146 & 0.0018 & 0.0134 & 0.0016 \\
\hline 5 & $\mathrm{a}$ & 0.0224 & 0.0022 & 0.0223 & 0.0022 \\
\hline 5 & b & 0.0229 & 0.0023 & 0.0188 & 0.0018 \\
\hline
\end{tabular}

the adsorption energies of the structures $2 \mathrm{~b}(170 \mathrm{meV})$ and $2 \mathrm{c}$ (168 meV). This can be understood considering that in $2 \mathrm{a}$ the resulting dipole moments are separated by the largest distance comparing to $2 \mathrm{~b}$ and $2 \mathrm{c}$. In turn, as the clusters grow, this electrostatic effect becomes less important. For example, the adsorption energies of the intercalated trimers (with smaller resulting dipole moment in comparison to the water dimer) and tetramers (with no resulting dipole moment) are closer. The adsorption energy increases a little for $\left(\mathrm{H}_{2} \mathrm{O}\right)_{5} \cdots \mid \cdot \cdots\left(\mathrm{H}_{2} \mathrm{O}\right)_{5}$ in structure $5 \mathrm{a}(177 \mathrm{meV}$ with PW91), but decreases in structure $5 b(157 \mathrm{meV})$.

In general, these intercalated systems appear to be more strongly bound than the one-side adsorbed systems analyzed in Sec. 3. However, the adsorption energy per molecule is in the same range obtained for the $\left(\mathrm{H}_{2} \mathrm{O}\right)_{n} \cdots \mid$ systems, as would be expected for pure GGA calculations. This result is also important 


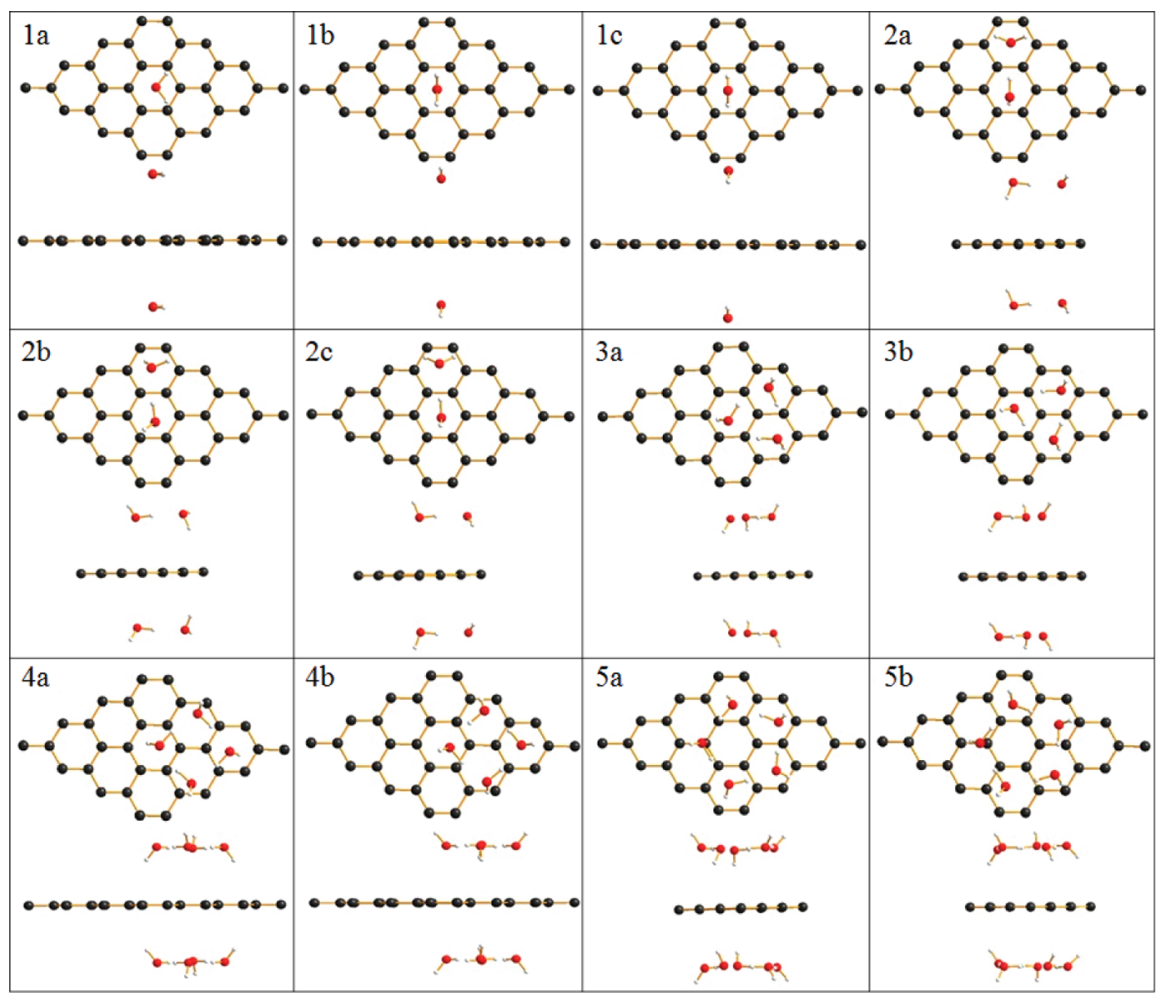

Figure 6. Structures of different water clusters intercalated by a graphene sheet. View from the top and side of all of the different adsorbates on the graphene surface.

to reinforce here that the energy related to the dipole interactions involving water molecules of distinct supercells does not affect the calculations of total energies in these systems. Again, the calculated interaction energies of the systems appear to be strongly influenced by the number of hydrogen bond formed in the aggregates. For this reason, we obtained small values (between 16 and $18 \mathrm{meV}$ with PW91) for the adsorption energy per molecules in the case of the adsorbed pentamers. For comparison, the adsorption energies calculated for the intercalated water monomers are similar $(35-36 \mathrm{meV})$ to those values obtained for $\mathrm{H}_{2} \mathrm{O} \cdots \mid$ (Table 3 ), which are still around $50 \%$ of the experimental estimates. ${ }^{26}$

4.2. Charge Transfer. In a similar fashion as obtained for the one-side adsorbed clusters, the calculated CT for the $\left(\mathrm{H}_{2} \mathrm{O}\right)_{n} \cdots$ | $\cdots\left(\mathrm{H}_{2} \mathrm{O}\right)_{n}$ systems increases with the growing number of water molecules in the aggregate. Unless structures $1 \mathrm{a}$ and $1 \mathrm{~b}$, that correspond to those where the $\mathrm{O}$ atoms are pointing toward the surface, all of the intercalated systems show CT from graphene to the water molecules. The Bader analysis obtained with both PBE and PW91 are presented in Table 9. Again, the PW91 scheme yields similar values for $\mathrm{CT}$ in comparison to PBE. Also, we noticed that in the case of single $\mathrm{H}_{2} \mathrm{O}$ intercalated by graphene, the direction of $\mathrm{CT}$ seems to depend on the orientation of the molecule at both sides (Figure 6). For example, considering structures $1 \mathrm{a}$ and $1 \mathrm{~b}$ of $\left(\mathrm{H}_{2} \mathrm{O}\right) \cdots \cdot \cdot \cdot\left(\mathrm{H}_{2} \mathrm{O}\right)$ the CT occurs from the molecules to graphene. On the other hand, in $1 \mathrm{c}$, we obtain a small inversion. Although in the aggregates intercalated by a single graphene layer the CT values are almost twice those obtained for the $\left(\mathrm{H}_{2} \mathrm{O}\right)_{n} \cdots \mid$ systems, there is no significant change close to the Fermi level, as displayed in the calculated DOS (Figure 7.). However, we noticed a significant increase of the CT to the water molecules in the intercalated dimers. This

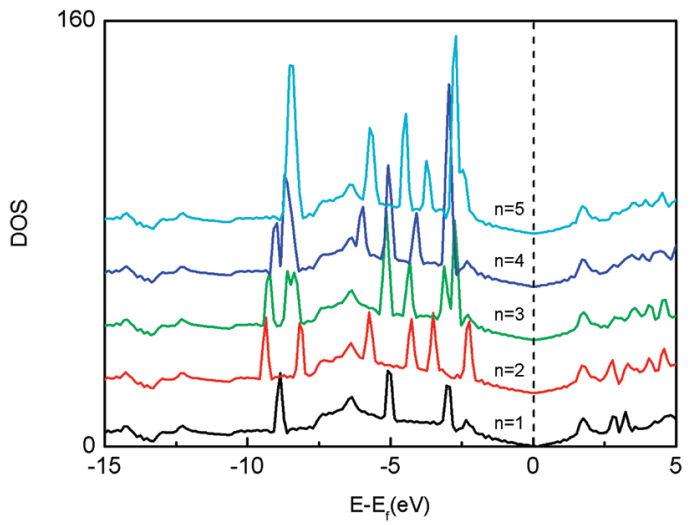

Figure 7. Calculated density of states (DOS) of different water clusters intercalated by a graphene sheet in the most stable orientations. The number of water molecules in the cluster is denoted by $n$.

effect can be related with the precise control of the amount of water adsorbed on the graphene surface in the experiments of opening the bandgap of graphene in an environmental chamber. ${ }^{9}$

\section{CONCLUSIONS}

In this work, we have performed a systematic study of small water aggregates adsorbed on a graphene surface within standard procedures of periodic first-principles DFT calculations. Our results showed that the small interactions of these adsorbates (in the range of 0.05 to $0.1 \mathrm{eV}$, with PW91-PAW) yield only mild effects in the electronic structure of graphene. In turn, the structuring of the aggregates near the surface is also slightly affected. Although the calculated adsorption energies do not 
completely describe the water-graphene interactions, the present methods have the advantage of using the PAW basis sets with a large plane wave cutoff and periodic supercells. Thus, we have avoided (i) the BSSE effects that are commonly introduced by localized basis sets and (ii) the effects of size and edge of the surface.

Pure GGA schemes underestimate the adsorption energies, which may be corrected by explicitly including the van der Waals interactions in the exchange-correlation potentials. However, we highlight that the use of USPP combined with PW91 can significantly increase the interaction energies between water and graphene. Despite its intrinsic difficulties with dispersion interactions, the PW91 functional may be a viable alternative to investigate the adsorption of large molecular aggregates or very large molecules on a graphene surface. Compared to experimental data and more accurate quantum-chemistry calculations, the present PW91-PAW adsorption energies are within around $50 \%$ of the best estimates. More importantly, these DFT methods yield dissociation curves for the $\mathrm{H}_{2} \mathrm{O}$-graphene systems presenting well-defined energy minima, although lacking long-range electron correlation.

We have found that in the case of the water-graphene-water systems the dipole orientation of the intercalated water clusters can play an important role in the wetting of graphene, considering the regime of small adsorbates.However, this effect tends to decrease as the clusters grow. Our calculated adsorption energies for these systems are in the $0.05-0.14 \mathrm{eV}$ range with $\mathrm{PBE}$ and 0.07-0.17 eV range with PW91. Generally, the $\left(\mathrm{H}_{2} \mathrm{O}\right)_{n} \cdots$ | $\cdots\left(\mathrm{H}_{2} \mathrm{O}\right)_{n}$ systems appear to be more strongly bound than the one-side adsorbed systems analyzed, but the adsorption energy per molecule is in the same range, as expected for pure GGA calculations. Considering the CT mechanism, we have noticed that it should preferentially occur from water to graphene only when the $\mathrm{O}$ atoms are pointing toward the surface. Otherwise, and in the case of larger adsorbed water clusters, the charge transfers systematically occur from graphene to water. Although the CT gives only a mild value, which is partially due to the calculation method, these results seem to be independent of the method of charge analysis.

\section{AUTHOR INFORMATION}

\section{Corresponding Author}

*E-mail: rivelino@ufba.br.

\section{ACKNOWLEDGMENT}

The authors acknowledge Conselho Nacional de Desenvolvimento Científico e Tecnológico (CNPq), Fundação CAPES and Fundação de Amparo à Pesquisa do Estado da Bahia (FAPESB) Brazilian agencies, for the support.

\section{REFERENCES}

(1) Xu, K.; Cao, P.; Heath, J. R. Science 2010, 329, 1188-1191.

(2) Booth, T. J.; Blake, P.; Nair, R. R.; Jiang, D.; Hill, E. W.; Bangert, U.; Bleloch, A.; Gass, M.; Novoselov, K. S.; Katsnelson, M. I.; et al. Nano Lett. 2008, 8, 2442-2446.

(3) Fileti, E. E.; Dalpian, G. M.; Rivelino, R. J. Appl. Phys. 2010, 108, 113527.

(4) Bunch, J. S.; Verbridge, S. S.; Alden, J. S.; van der Zande, A. M.; Parpia, J. M.; Craighead, H. G.; McEuen, P. L. Nano Lett. 2008, $8,2458-2462$.
(5) Santucci, S.; Picozzi, S.; Di Gregorio, F.; Lozzi, L.; Cantalini, C.; Valentini, L.; Kenny, J. M.; Delley, B. J. Chem. Phys. 2003, 119, 10904.

(6) Zhou, S. Y.; Siegel, D. A.; Fedorov, A. V.; Lanzara, A. Phys. Rev. Lett. 2008, 101, 086402.

(7) Leenaerts, O.; Partoens, B.; Peeters, F. M. Phys. Rev. B 2008, 77,125416

(8) Sabio, J.; Seoánez, C.; Fratini, S.; Guinea, F.; Neto, A. H. C.; Sols, F. Phys. Rev. B 2008, 77, 195409.

(9) Yavari, F.; Kritzinger, C.; Gaire, C.; Song, L.; Gulapalli, H.; Borca-Tasciuc, T.; Ajayan, P. M.; Koratkar, N. Small 2010, 6, 2535-2538.

(10) Neto, A. H. C.; Guinea, F.; Peres, N. M. R.; Novoselov, K. S.; Geim, A. K. Rev. Mod. Phys. 2009, 81, 109.

(11) Pontes, R. B.; Fazzio, A.; Dalpian, G. M. Phys. Rev. B 2009, 79, 033412.

(12) Medeiros, P. V. C.; Mascarenhas, A. J. S.; Mota, F. B.; de Castilho, C. M. C. Nanotechnology 2010, 48, 485701.

(13) Medeiros, P. V. C.; Mota, F. B.; Mascarenhas, A. J. S.; de Castilho, C. M. C. Nanotechnology 2010, 21, 115701.

(14) Berashevich, J.; Chakraborty, T. Phys. Rev. B 2009, 80, 033404.

(15) Ohta, T.; Bostwick, A.; Seyller, T.; Horn, K.; Rotenberg, E. Science 2006, 313, 951-954.

(16) Wehling, T. O.; Lichtenstein, A. I.; Katsnelson, M. I. Appl. Phys. Lett. 2008, 93, 202110.

(17) Schedin, F.; Geim, A. K.; Morozov, S. V.; Hill, E. W.; Blake, P.; Katsnelson, M. I.; Novoselov, K. S. Nat. Mater. 2007, 6, 652-655.

(18) Novoselov, K. S.; Geim, A. K.; Morozov, S. V.; Jiang, D.; Zhang, Y.; Dubonos, S. V.; Grigorieva, I. V.; Firsov, A. A. Science 2004, 306, 666-669.

(19) Leenaerts, O.; Partoens, B.; Peeters, F. M. Phys. Rev. B 2009, $79,235440$.

(20) Lin, C. S.; Zhang, R. Q.; Lee, S. T.; Elstner, M.; Frauenheim, Th.; Wan, L. J. J. Phys. Chem. B 2005, 109, 14183-14188.

(21) Xu, S.; Irle, S.; Musaev, D. G.; Lin, M. C. J. Phys. Chem. A 2005, 109, 9563-9572.

(22) Rubes, M.; Nachtigall, P.; Vondrasek, J.; Bludsky, O. J. Phys Chem. C 2009, 113, 8412-8419.

(23) Jeness, G. R.; Jordan, K. D. J. Phys. Chem. C 2009, 113, $10242-10248$

(24) Zhanpeisov, N. U.; Zhidomirov, G. M.; Fukumura, H. J. Phys. Chem. C 2009, 113, 6118-6123.

(25) Voloshina, E.; Usvyat, D.; Schütz, M.; Dedkov, Y.; Paulus, B. Phys. Chem. Chem. Phys. 2011, 13, 12041-12047.

(26) Helmy, A. K.; Ferreiro, E. A.; Bussetti, S. G. Appl. Surf. Sci. 2006, 253, 4966-4969.

(27) Perdew, J. P.; Chevary, J. A.; Vosko, S. H.; Jackson, K. A.; Pederson, M. R.; Singh, D. J.; Fiolhais, C. Phys. Rev. B 1992, 46, 6671.

(28) Perdew, J. P.; Burke, K.; Ernzerhof, M. Phys. Rev. Lett. 1996, $77,3865$.

(29) Perdew, J. P.; Wang, Y. Phys. Rev. B 1992, 45, 13244.

(30) Kresse, G.; Hafner, J. J. Phys.: Condens. Matter 1994, 6, 8245.

(31) Kresse, G.; Hafner, J. Phys. Rev. B 1993, 47, 558.

(32) Kresse, G.; Hafner, J. Phys. Rev. B 1994, 49, 14251.

(33) Kresse, G.; Furthmüller, J. Comput. Mater. Sci. 1996, 6, 15-50.

(34) G. Kresse, G.; J. Futhmüller, J. Phys. Rev. B 1996, 54, 11169.

(35) P. E. Blöchl, P. E. Phys. Rev. B 1994, 50, 17953.

(36) Kresse, G.; Joubert, D. Phys. Rev. B 1999, 59, 1758.

(37) Vanderbilt, D. Phys. Rev. B 1990, 41, 7892.

(38) Becke, A. D.; Johnson, E. R. J. Chem. Phys. 2007, 127, 154108.

(39) Kannemann, F. O.; Becke, A. D. J. Chem. Theory Comput. 2009, $5,719-727$.

(40) Monkhorst, H. J.; Pack, J. D. Phys. Rev. B 1976, 13, 5188.

(41) Tang, W.; Sanville, E.; Henkelman, G. J. Phys.: Condens. Matter 2009, 21, 084204.

(42) Császár, A. G.; Czakó, G.; Furtenbacher, T.; Tennyson, J.; Szalay, V.; Shirin, S. V.; Zobov, N. F.; Polyansky, O. L. J. Chem. Phys. 2005, 122, 214305.

(43) Gregory, J. K.; Clary, D. C.; Liu, K.; Brown, M. G.; Saykally, R. J. Science 1997, 275, 814-817. 
(44) The average distance between $\mathrm{H}_{2} \mathrm{O}$ and the graphene surface is calculated by subtracting weighted averages of the $\mathrm{H}_{2} \mathrm{O}$ atoms and the carbon atoms of the graphene sheet. The atomic number of the atoms was used as the weight factor in accordance with ref. (7).

(45) Tsuzuki, S.; Lüthi, H. P. J. Chem. Phys. 2001, 114, 3949. 